\title{
RESPOSTAS DE CULTIVARES DE SOJA A HERBICIDAS
}

\author{
Erivelton S. Roman ${ }^{1}$, Fábio Tosso ${ }^{2}$ e José Antônio A. Marinho ${ }^{2}$
}

Embrapa Trigo. Caixa Postal 451. Passo Fundo, RS 99001-970 eroman@cnpt.embrapa.br
2Faculdade de Agronomia e Medicina Veterinária da Universidade de Passo Fundo. Passo Fundo, RS

\section{RESUMO}

Um experimento foi conduzido em Passo Fundo, RS, em um Latossolo Vermelho distrófico típico, durante a safra 1999/ 2000, para avaliar diferenças entre cultivares de soja quanto às suas tolerâncias ou susceptibilidades a herbicidas aplicados isolados ou em misturas. Reduções na altura da soja foram observados na cultivar BRS 153 e na BRS 138 em relação à testemunha. A cultivar BRS 153 teve menor altura apenas no tratamento com diclosulan a $70 \mathrm{~g} /$ ha, enquanto a cultivar BRS 138, apresentou menor altura nos tratamentos com sulfentrazone a $1200 \mathrm{~g} / \mathrm{ha}$, diclosulan a $70 \mathrm{~g} / \mathrm{ha}$, sulfentrazone+diclosulan a $250+25 \mathrm{~g} / \mathrm{ha}$ e sulfentrazone+clomazone a 1000+1440 g/ha. Não foram detectados efeitos significativos dos tratamentos químicos na densidade de plantas das diferentes cultivares e nem sobre a produtividade de grãos de soja.

Palavras-chave: sulfentrazone, diclosulan, clomazone, imazethapyr.

\section{ABSTRACT \\ Responses of soybean cultivars to herbicides}

An experiment was conducted in Passo Fundo, RS, on a Red Oxissol distrofic soil, during the 1999/2000 soybean growing season to evaluate cultivar differences in response to herbicides applied alone and as tank mixtures. Height reductions were observed in cultivars BRS 153 and BRS 138. The cultivar BRS 153 exhibited height reductions to diclosulan applied at the rate of $70 \mathrm{~g} / \mathrm{ha}$ while the cultivar BRS 138 exhibited height reductions to sulfentrazone at $1200 \mathrm{~g} / \mathrm{ha}$, diclosulan at $70 \mathrm{~g} / \mathrm{ha}$, sulfentrazone + diclosulan at $250+25 \mathrm{~g} / \mathrm{ha}$ and sulfentrazone + clomazone at $1000+1440 \mathrm{~g} / \mathrm{ha}$. There was no effect of the treatmens on the other evaluated soybean parameters, including yield.

Key words: sulfetrazone, diclosulan, clomazone, imazethapyr.

\section{INTRODUÇÃO}

Têm sido relatados, no Brasil, vários casos de resistência de plantas daninhas a herbicidas cujo mecanismo de ação é a inibição da enzima ALS (Christoffoleti et al., 1994; Vidal \& Fleck, 1997), devido ao repetido uso de produtos químicos que atuam nesse sítio bioquímico. O uso de herbicidas, com diferentes mecanismos de ação e rotas de degradação metabólica, é sugerido como uma estratégia para evitar ou retardar o aparecimento do problema (Gressel \& Segel, 1990).
O uso de herbicidas cujo mecanismo de ação é na inibição da enzima protoporfirina IX (protox), uma das enzimas que atuam na síntese de clorofila, é alternativa para o manejo da resistência aos inibidores da ALS. Esses herbicidas causam acumulação de protox que, em presença de luz e de oxigênio molecular, gera oxigênio elementar, o qual causa destruição da membrana celular, rápida dessecação e necrose de tecidos. Possuem modo de ação essencialmente de contato (Lydon \& Duke, 1988). Não são conhecidas espécies resistentes aos herbicidas inibidores da protox (Duke et al., 1996), de forma 
que esses herbicidas podem ser importantes em programas de manejo de plantas daninhas.

Sulfentrazone é um herbicida inibidor de protox (Dayan et al., 1997). A característica deste composto é que, diferentemente de outros produtos com mecanismo e modo de ação semelhante, possue atividade pré-emergente (Dayan et al., 1996). No entanto, as cultivares de soja variam em sua tolerância ao produto, quando aplicado em pré-emergência (Walker et al., 1992). O metabolismo de sulfentrazone é o fator mais importante na tolerância da soja a esse herbicida, determinando diferenças entre cultivares em relação a essa característica (Dayan et al., 1997).

São relatados danos à soja quando o produto é usado em solos com baixos teores de matéria orgânica e de argila, de forma que são necessários estudos para verificar a tolerância ou a susceptibilidade de algumas variedades de soja ao sulfentrazone.

Os objetivos deste trabalho foram:

1) De avaliar a resposta de algumas cultivares de soja ao herbicida sulfentrazone, aplicado em pré-emergência, isolado ou em mistura com outros herbicida.

2) Determinar a tolerância de algumas cultivares de soja a alguns herbicidas registrados para uso na cultura.

\section{MATERIAL E MÉTODOS}

Um experimento foi conduzido em condições de campo na área experimental da Embrapa Trigo, localizada no município de Coxilha, RS, no ano agrícola de 1999/2000. O solo é de textura média, com $44 \%$ da argila e 4,2\% de matéria orgânica, pertencente à Unidade de Mapeamento Passo Fundo (Latossolo Vermelho típico distrófico).

Dez cultivares de soja foram selecionadas com base nas suas áreas semeadas nos Estados do Rio Grande do Sul e de Santa Catarina (Anônimo, 1999), para determinar-se as suas respostas à aplicação de sulfentrazone, aplicado isolado e em mistura com outros herbicidas, em pré-emergência. Um tratamento com imazetapyr, aplicado em pós-emergência, foi também testado. Doses elevadas de sulfentrazone e dos demais herbicidas foram também usadas para verificar a tolerância das mesmas, quando houvesse, em condições de campo, sobrepasse da barra de aplicação, dobrando a dose normalmente recomendada para o controle das plantas daninhas. $\mathrm{O}$ delineamento experimental foi em blocos ao acaso, em esquema de blocos em faixa, em fatorial. Os fatores foram variedades de soja (Tabela 1) e herbicidas (Tabela 2), com quatro repetições. Os herbicidas foram aplicados nas parcelas, as quais mediam 3,0 $\mathrm{m} \times 36 \mathrm{~m}$. As sub-parcelas (nas faixas) mediam 3,0 $m \times 3,6 m$ e eram compostas pelas variedades.

As cultivares de soja reagentes encontram-se discriminadas na Tabela 1 e foram semeadas no espaçamento de $0,45 \mathrm{~m}$ entre as linhas. A semeadura foi realizada no dia $16 \mathrm{de}$ novembro de 1999, em sistema de plantio direto. A adubação foi realizada usando-se $250 \mathrm{~kg} /$ ha de adubo, fórmula $05-25-25$.

Tabela 1. Variedades utilizadas no experimento. Embrapa Trigo, Passo Fundo, RS.1999/ 2000.

\begin{tabular}{ll}
\hline Variedades & Ciclo das Variedades \\
\hline CD 201 & Médio \\
Embrapa 66 & Médio \\
BRS 137 & Precoce \\
BRS 153 & Médio \\
Embrapa 48 & Precoce \\
BR 37 & Médio \\
RS 10 & Semitardio/tardio \\
RS 5 & Semitardio/tardio \\
BR 16 & Médio \\
BRS 138 & Precoce \\
\hline
\end{tabular}

Os tratamentos em pré-emergência (Tabela 2) foram aplicados no dia 20/11/99, com pulverizador costal de precisão, munido de bicos de jato em forma de leque, do tipo 110. 015 , a $1,1 \mathrm{~kg} / \mathrm{cm}^{2}$ de pressão de trabalho, dada por gás carbônico, e consumo de calda de 100 l/ha. A pulverização foi realizada com início às 09:10 e término às 10:40 h. No momento da pulverização dos tratamentos o solo encontrava-se úmido, a umidade relativa do ar era de $65 \%$ e a temperatura ambiente $27^{\circ} \mathrm{C}$. Precipitações pluviais totalizando $6,3 \mathrm{~mm}$ ocorreram nos 5 dias posteriores à aplicação dos tratamentos de pré-emergência. O tratamento em pós-emergência (Tabela 2) foi pulverizado no dia 10/01/ 2000 , com o mesmo equipamento e calibração já descritos. A pulverização foi realizada com início as 18:00 h e término as 18:15 h. Naquela ocasião a umidade relativa do ar era de $70 \%$, com temperatura ambiente de $27,5^{\circ} \mathrm{C}$. O solo encontrava-se úmido, tendo ocorrido uma precipitação pluvial de 32,4 $\mathrm{mm}$ no dia anterior (Tabela 3 ).

Foram determinadas a altura média de 5 plantas por subparcela, no florescimento pleno das variedades. A densidade de plantas foi determinada por ocasião da colheita, contando-se o número de plantas por metro linear, em 3 amostragens por subparcela. $O$ rendimento de grãos (peso de grãos com $13 \%$ de umidade) foi determinado colhendo-se uma área de $10 \mathrm{~m}^{2}$ por parcela e transformados em quilogramas por hectare.

Após a análise da variância, as médias dos tratamentos foram comparadas com a testemunha capinada, pelo teste de Dunnett ao nível de 5\% de probabilidade de erro, usandose o programa SAS (proc glm). 
Tabela 2. Produtos usados no experimento. Embrapa Trigo, Passo Fundo, RS. 1999-2000.

\begin{tabular}{|c|c|c|c|c|}
\hline \multicolumn{2}{|c|}{ Herbicidas } & \multicolumn{2}{|c|}{ Dose } & \multirow{2}{*}{ Época de aplicação } \\
\hline Nome comum & Nome comercial & g/ha i.a. & g ou l/ha p.c. & \\
\hline Sulfentrazone & Boral $500 \mathrm{SC}$ & 600 & 1,21 & Pré-emergência \\
\hline Sulfentrazone & Boral $500 \mathrm{SC}$ & 1200 & 2,41 & Pré-emergência \\
\hline Diclosulan & Spider & 35 & $42 \mathrm{~g}$ & Pré-emergência \\
\hline Diclosulan & Spider & 70 & $84 \mathrm{~g}$ & Pré-emergência \\
\hline Sulfentrazone + diclosulan & Boral $500 \mathrm{SC}+$ Spider & $250+25$ & $0,5 \mathrm{l}+30 \mathrm{~g}$ & Pré-emergência \\
\hline Sulfentrazone+diclosulan & Boral $500 \mathrm{SC}+$ Spider & $500+50$ & $1,0 \mathrm{I}+60 \mathrm{~g}$ & Pré-emergência \\
\hline Sulfentrazone+clomazone & Boral $500 \mathrm{SC}+$ Gamit $360 \mathrm{CS}$ & $500+720$ & $1,01+2,01$ & Pré-emergência \\
\hline Sulfentrazone + clomazone & Boral $500 \mathrm{SC}+$ Gamit $360 \mathrm{CS}$ & $1000+1440$ & $2,01+4,01$ & Pré-emergência \\
\hline Imazethapyr & Pivot & 100 & 1,01 & Pós-emergência \\
\hline
\end{tabular}

Tabela 3. Precipitações pluviais observadas antes e após a instalação do experimento. Embrapa Trigo, Passo Fundo, RS. 1999/2000.

\begin{tabular}{|c|c|c|c|}
\hline \multirow{2}{*}{ Dia } & \multicolumn{3}{|c|}{ Precipitação ( $\mathrm{m}$ m) } \\
\hline & Novem bro & Dezem bro & Janeiro \\
\hline 1 & 0,0 & 0,0 & 0,0 \\
\hline 2 & 9,2 & 0,0 & 0,0 \\
\hline 3 & 21,0 & 18,4 & 0,0 \\
\hline 4 & 0,0 & 11,0 & 0,0 \\
\hline 5 & 42,4 & 0,0 & 0,0 \\
\hline 6 & 0,2 & 26,8 & 1,0 \\
\hline 7 & 0,0 & 0,0 & 0,0 \\
\hline 8 & 0,0 & 32,4 & 0,0 \\
\hline 9 & 2,1 & 0,0 & 0,0 \\
\hline 10 & 0,0 & 0,0 & $3,0 * *$ \\
\hline 11 & 0,0 & 0,0 & 21,9 \\
\hline 12 & 0,0 & 0,0 & 6,3 \\
\hline 13 & 3,2 & 26,2 & 0,0 \\
\hline 14 & 27,2 & 9,8 & 0,0 \\
\hline 15 & 0,0 & 0,0 & 23,2 \\
\hline 16 & 0,0 & 0,0 & 24,4 \\
\hline 17 & 0,0 & 0,0 & 17,0 \\
\hline 18 & 0,0 & 0,0 & 16,0 \\
\hline 19 & 0,0 & 0,0 & 0,0 \\
\hline 20 & $3,3 *$ & 0,2 & 0,0 \\
\hline 21 & 3,7 & 0,0 & 0,0 \\
\hline 22 & 0,0 & 0,7 & 0,0 \\
\hline 23 & 0,0 & 0,0 & 0,0 \\
\hline 24 & 0,0 & 0,0 & 0,0 \\
\hline 25 & 4,4 & 0,0 & 22,8 \\
\hline 26 & 0,0 & 0,0 & 0,0 \\
\hline 27 & 1,9 & 0,0 & 0,0 \\
\hline 28 & 0,0 & 0,0 & 0,0 \\
\hline 29 & 0,0 & 0,0 & 0,0 \\
\hline 30 & 0,0 & 5,6 & 0,0 \\
\hline 31 & - & 0,0 & 8,0 \\
\hline Total & 118,6 & 131,1 & 143,6 \\
\hline Normal & 141,4 & 161,5 & 143,4 \\
\hline
\end{tabular}

* Dia da aplicação dos tratamentos em pré-emergência.

** Dia da aplicação dos tratamentos em pós-emergência.

\section{RESULTADOS E DISCUSSÃO}

As interações entre variedades e tratamentos na altura de plantas e no rendimento de grãos foram significativas $(\mathrm{P}<$ $0.0001)$. Reduções da altura de plantas de soja foram observados na cultivar BRS 153 e na BRS 138 em relação à testemunha (Tabela 4). A cultivar BRS 153 teve menor altura apenas no tratamento com diclosulan a $70 \mathrm{~g} / \mathrm{ha}$, enquanto a cultivar BRS 138 apresentou menor altura nos tratamentos com sulfentrazone a $1200 \mathrm{~g} / \mathrm{ha}$, diclosulan a $70 \mathrm{~g} / \mathrm{ha}$, sulfentrazone+diclosulan a $250+25$ $\mathrm{g} /$ ha e sulfentrazone + clomazone a $1000+1440 \mathrm{~g} / \mathrm{ha}$. Por exemplo, a altura da cultivar BRS 153 foi suprimida em 14,7\% por diclosulan a $70 \mathrm{~g} /$ ha, enquanto a altura da cultivar BRS 138 foi reduzida em $16,5 \%$ por sulfentrazone a $1200 \mathrm{~g} / \mathrm{ha}$. A redução da altura das plantas, nesses tratamentos, foi indicação da menor tolerância dessas cultivares, embora essas doses testadas fossem elevadas (dobro da dose recomendada), mas não foram detectados outros sintomas de fitotoxicidade à soja.

Não foram detectados efeitos significativos dos tratamentos químicos na densidade de plantas das diferentes cultivares (Tabela 4). Dayan et al. (1996) demonstraram que sulfentrazone não inibiu a germinação de Senna obtusifolia L. e de Cassia occidentalis L., a despeito da extrema sensibilidade dessas espécies ao herbicida, sugerindo que a morte de plântulas de espécies sensíveis ao produto ocorre pela absorção de sulfentrazone via radicular. As condições que favorecem a ação fitotóxica de sulfentrazone (e de vários outros herbicidas residuais) são as de solo com baixos teores de matéria orgânica e com elevados teores de umidade (Wehtje et al., 1995), com a concentração de herbicida na solução do solo aumentando com o acréscimo do teor de areia e com a diminuição do teor de argila (Grey et al., 1996). Como o solo onde o experimento foi conduzido é de textura média, com ocorrência de baixas precipitações pluviais durante os processos de germinação e emergência da soja (Tabela 3), é provável que as quantidades de produtos disponíveis na solução do solo e absorvidos não tenham sido suficientes para reduzir a população de plantas de soja no experimento. 
Tabela 4. Efeito dos tratamentos na altura (cm) das plantas de soja. Embrapa Trigo, Passo Fundo, RS. $1999 / 2000$.

\begin{tabular}{|c|c|c|c|c|c|c|c|c|c|c|}
\hline \multirow[b]{2}{*}{ TRATAMENTO } & \multicolumn{10}{|c|}{ VARIEDADE } \\
\hline & $\begin{array}{c}\text { Codetec } \\
201\end{array}$ & $\begin{array}{c}\text { Embrapa } \\
66\end{array}$ & $\begin{array}{c}\text { BRS } \\
137\end{array}$ & $\begin{array}{c}\text { BRS } \\
153\end{array}$ & $\begin{array}{c}\text { Embrapa } \\
48\end{array}$ & $\begin{array}{c}\text { BR } \\
37\end{array}$ & $\begin{array}{c}\text { RS } \\
10\end{array}$ & $\begin{array}{c}\mathrm{RS} \\
5\end{array}$ & $\begin{array}{c}\mathrm{BR} \\
16\end{array}$ & $\begin{array}{c}\text { BRS } \\
138 \\
\end{array}$ \\
\hline Testemunha capinada & 64,7 & 67,7 & 63,5 & 65,7 & 70,5 & 72,2 & 73,2 & 63,5 & 82,2 & 62,5 \\
\hline Sulfentrazone (600 g/ha) & 74,5 & 72,2 & 64,7 & 60,2 & 71,5 & 72,5 & 71,5 & 68,0 & 81,5 & 53,5 \\
\hline Sulfentrazone ( $1200 \mathrm{~g} / \mathrm{ha})$ & 71,0 & 71,7 & 56,0 & 63,2 & 72,5 & 75,7 & 73,2 & 67,5 & 83,2 & $52,2 *$ \\
\hline Diclosulan $(35 \mathrm{~g} / \mathrm{ha})$ & 78,2 & 67,7 & 70,2 & 65,0 & 68,5 & 74,5 & 73,7 & 63,7 & 82,5 & 56,5 \\
\hline Diclosulan (70 g/ha) & 73,2 & 66,0 & 63,2 & $56,0 *$ & 66,7 & 72,2 & 68,2 & 60,7 & 80,0 & $48,2 *$ \\
\hline Sulfentrazone+diclosulan $(250+25 \mathrm{~g} / \mathrm{ha})$ & 76,0 & 70,5 & 73,0 & 63,5 & 68,0 & 73,5 & 70,7 & 66,2 & 83,7 & $50,5 *$ \\
\hline Sulfentrazone+diclosulan $(500+50 \mathrm{~g} / \mathrm{ha})$ & 72,5 & 67,5 & 68,0 & 60,0 & 66,5 & 73,7 & 68,7 & 65,5 & 82,7 & 55,0 \\
\hline Sulfentrazone+clomazone $(500+720 \mathrm{~g} / \mathrm{ha})$ & 74,0 & 71,7 & 71,5 & 60,5 & 68,0 & 78,2 & 73,2 & 68,7 & 83,7 & 58,5 \\
\hline Sulfentrazone+clomazone $(1000+1440 \mathrm{~g} / \mathrm{ha})$ & 72,2 & 70,5 & 67,5 & 63,7 & 67,7 & 76,0 & 70,7 & 67,2 & 83,5 & $51,0 *$ \\
\hline Imazethapyr (100 g/ha) & 60,0 & 61,5 & 66,0 & 60,7 & 64,2 & 71,2 & 66,2 & 64,7 & 79,0 & 59,0 \\
\hline DMS $5 \%$ & 15,0 & 8,5 & 13,4 & 7,9 & 9,2 & 6,9 & 7,5 & 7,6 & 9,2 & 9,0 \\
\hline
\end{tabular}

Médias seguidas por asterisco são estatisticamente inferiores à testemunha capinada, pelo teste de Dunnett ao nível de probabilidade de erro de 5 \%.

Apesar de alguns tratamentos químicos terem afetado a altura de plantas em algumas variedades (Tabela 5), as produtividades de grãos das cultivares não foram reduzidas pelos tratamentos químicos (Tabela 6). A habilidade das cultivares em metabolizar os produtos testados pode explicar a ausência de efeito dos tratamentos químicos sobre esse parâmetro, pois o metabolismo rápido é a base da tolerância da soja ao sulfentrazone e a herbicidas inibidores do protox e da ALS (Kent et al., 1988; Wixson \& Shaw, 1991; EINaggar et al., 1992; Dayan et al., 1997). Além disso, a textura média e o teor relativamente elevado de matéria orgânica do solo no qual o experimento foi conduzido, explica, em parte, a inexistência de efeitos dos tratamentos herbicidas sobre a produtividade de grãos de soja. Solos com baixos teores de matéria orgânica e com elevados teores de umidade, propiciam teores elevados de sulfentrazone na solução do solo, favorecendo os danos causados por esse herbicida à soja (Wehtje et al., 1995), os quais aumentam com o teor de areia no solo (Grey et al., 1996) e, portanto, com menores teores de argila. O uso de sulfentrazone é recomendado somente em solos com teores de argila superiores a $30 \%$.

Tabela 5. Efeito dos tratamentos na densidade (número de indivíduos $/ \mathrm{m}^{2}$ ) de plantas de soja. Embrapa Trigo, Passo Fundo, RS. $1999 / 2000$.

\begin{tabular}{|c|c|c|c|c|c|c|c|c|c|c|}
\hline \multirow[b]{2}{*}{ TRATAMENTO } & \multicolumn{10}{|c|}{ VARIEDADE } \\
\hline & $\begin{array}{c}\text { Codetec } \\
201\end{array}$ & $\begin{array}{c}\text { Embrapa } \\
66\end{array}$ & $\begin{array}{c}\text { BRS } \\
137\end{array}$ & $\begin{array}{c}\text { BRS } \\
153\end{array}$ & $\begin{array}{c}\text { Embrapa } \\
48\end{array}$ & $\begin{array}{l}\mathrm{BR} \\
37\end{array}$ & $\begin{array}{l}\mathrm{RS} \\
10 \\
\end{array}$ & $\begin{array}{c}\mathrm{RS} \\
5\end{array}$ & $\begin{array}{c}\mathrm{BR} \\
16 \\
\end{array}$ & $\begin{array}{c}\text { BRS } \\
138 \\
\end{array}$ \\
\hline Testemunha capinada & 28 & 19 & 17 & 24 & 27 & 38 & 20 & 34 & 27 & 18 \\
\hline Sulfentrazone ( $600 \mathrm{~g} / \mathrm{ha})$ & 23 & 18 & 16 & 21 & 26 & 29 & 22 & 25 & 23 & 13 \\
\hline Sulfentrazone ( $1200 \mathrm{~g} / \mathrm{ha})$ & 17 & 16 & 14 & 20 & 19 & 32 & 17 & 36 & 26 & 17 \\
\hline Diclosulan (35 g/ha) & 19 & 19 & 17 & 23 & 29 & 35 & 24 & 38 & 23 & 24 \\
\hline Diclosulan (70 g/ha) & 23 & 18 & 21 & 21 & 24 & 26 & 20 & 32 & 28 & 13 \\
\hline Sulfentrazone+diclosulan $(250+25 \mathrm{~g} / \mathrm{ha})$ & 18 & 27 & 15 & 22 & 32 & 27 & 28 & 24 & 27 & 21 \\
\hline Sulfentrazone+diclosulan $(500+50 \mathrm{~g} / \mathrm{ha})$ & 21 & 17 & 14 & 20 & 31 & 28 & 24 & 25 & 32 & 18 \\
\hline Sulfentrazone+clomazone $(500+720 \mathrm{~g} / \mathrm{ha})$ & 23 & 19 & 20 & 23 & 18 & 37 & 22 & 30 & 26 & 18 \\
\hline Sulfentrazone+clomazone $(1000+1440 \mathrm{~g} / \mathrm{ha})$ & 19 & 21 & 23 & 18 & 20 & 26 & 23 & 33 & 23 & 22 \\
\hline Imazethapyr (100 g/ha) & 20 & 19 & 19 & 22 & 24 & 38 & 23 & 31 & 25 & 19 \\
\hline DMS $5 \%$ & 12 & 9 & 9 & 11 & 17 & 23 & 16 & 10 & 17 & 16 \\
\hline
\end{tabular}

Médias seguidas por asterisco são estatisticamente inferiores à testemunha, pelo teste de Dunnett ao nível de probabilidade de erro de 5 \%.

\section{CONCLUSÃO}

Os produtos avaliados são seletivos às variedades de soja testadas, em solos com teores médios de argila. Peque- nas diferenças em respostas aos tratamentos, observadas entre as cultivares, podem ser, em parte, atribuídas à habilidade intrínsica de cada cultivar em sobrepujar os estresses causados pelos tratamentos. 
Tabela 6. Efeito dos tratamentos no rendimento de grãos (kg/ha) de soja. Embrapa Trigo, Passo Fundo, RS. $1999 / 2000$.

\begin{tabular}{|c|c|c|c|c|c|c|c|c|c|c|}
\hline \multirow[b]{2}{*}{ TRATAMENTO } & \multicolumn{10}{|c|}{ VARIEDADE } \\
\hline & $\begin{array}{c}\text { Codetec } \\
201\end{array}$ & $\begin{array}{c}\text { Embrapa } \\
66\end{array}$ & $\begin{array}{c}\text { BRS } \\
137\end{array}$ & $\begin{array}{c}\text { BRS } \\
153\end{array}$ & $\begin{array}{c}\text { Embrapa } \\
48\end{array}$ & $\begin{array}{l}\mathrm{BR} \\
37 \\
\end{array}$ & $\begin{array}{c}\text { RS } \\
10 \\
\end{array}$ & $\begin{array}{c}\mathrm{RS} \\
5 \\
\end{array}$ & $\begin{array}{c}\text { BR } \\
16 \\
\end{array}$ & $\begin{array}{c}\text { BRS } \\
138 \\
\end{array}$ \\
\hline Testemunha capinada & 2279 & 2400 & 2082 & 2767 & 2106 & 2485 & 2541 & 2673 & 2625 & 2129 \\
\hline Sulfentrazone $(600 \mathrm{~g} / \mathrm{ha})$ & 2942 & 3230 & 2466 & 2919 & 2563 & 2732 & 2713 & 2842 & 2867 & 2185 \\
\hline Sulfentrazone (1200 g/ha) & 2875 & 3236 & 1899 & 3367 & 2707 & 2945 & 2825 & 2867 & 3118 & 2245 \\
\hline Diclosulan (35 g/ha) & 2997 & 2947 & 2834 & 2818 & 2387 & 2563 & 2644 & 2910 & 2750 & 2097 \\
\hline Diclosulan (70 g/ha) & 3192 & 2728 & 2856 & 2853 & 2228 & 2732 & 2625 & 2753 & 2847 & 1907 \\
\hline Sulfentrazone+diclosulan $(250+25 \mathrm{~g} / \mathrm{ha})$ & 3224 & 3088 & 2871 & 3171 & 2281 & 2728 & 2845 & 2976 & 2844 & 2088 \\
\hline Sulfentrazone + diclosulan $(500+50 \mathrm{~g} / \mathrm{ha})$ & 3262 & 2952 & 2906 & 3197 & 2533 & 2891 & 2811 & 2963 & 3024 & 2017 \\
\hline Sulfentrazone+clomazone $(500+720 \mathrm{~g} / \mathrm{ha})$ & 3081 & 3052 & 2508 & 3066 & 2400 & 2753 & 2834 & 3148 & 3021 & 2321 \\
\hline Sulfentrazone+clomazone $(1000+1440 \mathrm{~g} / \mathrm{ha})$ & 3078 & 3123 & 2643 & 3475 & 2492 & 2969 & 2929 & 3007 & 3033 & 1892 \\
\hline Imazethapyr (100 g/ha) & 2527 & 2597 & 2552 & 2986 & 2242 & 2546 & 2526 & 3046 & 2901 & 2590 \\
\hline DMS $5 \%$ & 494 & 571 & 629 & 540 & 652 & 457 & 482 & 544 & 652 & 633 \\
\hline
\end{tabular}

Médias seguidas por asterisco são estatisticamente diferentes da testemunha, pelo teste de Dunnett ao nível de probabilidade de erro de 5 \%.

\section{LITERATURA CITADA}

ANÔNIMO. Recomendações técnicas para a cultura da soja no Rio Grande do Sul e Santa Catarina 1999/00. REUNIÃO DE PESQUISA DE SOJA DA REGIÃO SUL, 27. Chapecó, SC. 167p. 1999.

CHRISTOFFOLETI, P. J.; VICTORIA FILHO, R.; SILVA, C. B. Resistência de plantas daninhas aos herbicidas. Planta Daninha, v. 12, n. 1, p. 13-20, 1994.

DAYAN, F. E.; WEETE, J. D. Mechanism of tolerance to a novel phenyl triazolinone herbicide. Proc. Am. Soc. Plant Physiology, v. 111, p. 119, 1996.

DAYAN, F. E.; DUKE, S. O.; REDDY, K. N.; HAMPER, B. C.; LESCHINSKI, K. L. Effect of isoxazoles on protoporphyrinogen oxidase and porphyrin physiology. J. Agric. Food Chemistry, v. 52, p. $967-$ 975, 1997a.

DAYAN, F. E.; WEETE, J. D.; DUKE, O. S.; HANCOCK, H. G. Soybean (Glycine max) cultivar diferences in response to sulfentrazone. Weed Science, v. 45, p. 634-641, $1997 \mathrm{~b}$.

DUKE, S. O.; DAYAN, F. E.; YAMAMOTO, M.; DUKE, M. V.; REDDY, K. N. Protoporphyrinogen oxidase inhibitors - their current and future role. PROC. INTERNATIONAL WEED CONTROL CONGRESS. 3: 775-780, 1996.

ELNAGGAR, S. E. R.; CREEKMORE, R. W.; SCHOKEN, M. J.; ROSEN, R. T.; ROBINSON, R. A. Metabolism of clomazone herbicide in soybean. J. Agric. Food Chemistry, v. 40, p. 880-883, 1992.
GRESSEL, J.; SEGEL, L. A. Modeling the effectiveness of herbicide rotations and mixtures as strategies to delay or preclude resistance. Weed Technology, v. 4, p. 186-198, 1990.

GREY, T. L.; WEHTJE, G. R.; WALKER, R. H. Sorption and mobility of sulfentrazone as affected by soil and $\mathrm{pH}$. PROCEEDINGS OF THE SOUTHERN WEED SCIENCE SOCIETY, 49: 176, 1996.

KENT, L. M.; BARRENTINE, W. L.; WILLS, G. D. Response of twenty determinate soybean (Glycine max) cultivars to imazaquin. PROCEEDINGS OF THE SOUTHERN WEED SCIENCE SOCIETY 41: 50, 1988.

LYDON, J.; DUKE, O. S. Porphyrin synthesis is required for photobleaching activity of the $p$-nitro substituted diphenyl ether herbicides. PESTIC. BIOCHEM. PHYSIOL.v. 36, p. 300-307, 1988.

VIDAL, R.; A. FLECK, N. G. Three weed species with confirmed resistance to herbicides in Brazil. In: MEETING OF THE WEED SCIENCE SOCIETY OF AMERICA, 37, 1997. Abstracts ... Champaign: WSSA, 1997, p. 100.

WALKER, R.H.; RICHBURG, J. S.; JONES, R. E. F6285 efficacy as affected by rate and method of application. PROCEEDINGS OF THE SOUTHEM WEED SCIENCE SOCIETY 45:51, 1992.

WEHTJE, G.; WALKER, R. H.; GREY, T. L. SPRATLIN, C. E. Soil effects of sulfentrazone. PROCEEDINGS OF THE SOUTHERN WEED SCIENCE SOCIETY, 50: 177-178, 1995.

WIXSON, M. B.; SHAW, D. R. Diferential response of soybean (Glycine max) cultivars to AC 263222. Weed Technology v. 5, p. 430-433, 1991. 
